\title{
Relación de las Habilidades Sociales con el rendimiento académico en estudiantes Universitarios
}

\section{Relationship of Social Skills with academic performance in University students}

\author{
Herbert Cosio, Miguel Núñez, Liceth Lazo ${ }^{3}$
}

\section{RESUMEN}

Él objetivo fue establecer la relación de las Habilidades Sociales y el Rendimiento Académico de los estudiantes universitarios. La investigación se desarrolló dentro del paradigma cuantitativo, se utilizó el método deductivo, de alcance relacional y analítico. La unidad de análisis estuvo constituido por estudiantes de la universidad Andina de Cusco. La muestra estuvo constituido por 201 estudiantes, mediante un muestreo probabilístico aleatorio simple. La prueba de hipótesis fue contratada mediante el estadístico paramétrico de correlación de Pearson. El resultado obtenido fue a mayores habilidades sociales, mayor el rendimiento académico en los estudiantes, se obtuvo una correlación de Pearson de 0,829 con un factor de determinación del 68,6\%. Se concluye que existe asociación directamente proporcional entre las Habilidades Sociales y rendimiento académico de los estudiantes de Medicina Humana de la Universidad Andina de Cusco.

Palabras clave: Rendimiento académico y Habilidades Sociales (DesC)

\section{ABSTRACT}

The objective was to establish the relationship of Social Skills and Academic Performance of university students. The research was developed within the quantitative paradigm, the deductive method, relational and analytical scope was used. The analysis unit consisted of students from the Andean University of Cusco. The sample consisted of 201 students, using a simple random probability sampling. The hypothesis test was hired using Pearson's parametric correlation statistic to analyze the two variables measured in interval. The result obtained was the higher the social skills, the higher the academic performance in the students, a Pearson correlation of 0.829 was obtained with a determining factor of $68.6 \%$. It is concluded that there is a directly proportional association between Social Skills and academic performance of Human Medicine students of the Andean University of Cusco.

Keywords: Academic performance and Social Skills (MeSH)

1. Cirujano Dentista UCSM, Magíster en Estomatología UPCH, Doctor en Educación UCSM, Docente a tiempo completo de la Universidad Alas Peruanas Filial Cusco

2. Médico Cirujano UNSAAC, Magister en Docencia y Gestión Educativa UAP

3. Cirujano Dentista UAC, Magister en Salud con mención en Salud Publica UANCV, Doctor es Odontología UAP, Docente a tiempo completo de la Universidad Alas Peruanas Filial Cusco 


\section{INTRODUCCIÓN}

Las habilidades sociales están recibiendo una especial atención en estos días, en función a los procesos de licenciamiento y acreditación que vienen sometiéndose las universidades en nuestro país. Estamos hablando del comportamiento social que un estudiante universitario tiene $y$ el cual lo ha desarrollado desde niño, probablemente en su primera infancia. Las habilidades sociales son importantes porque permite que los jóvenes asuman roles y normas sociales y les ayude a relacionarse dentro del salón de clases. Está muy asociado las habilidades que los jóvenes desarrollen con la adaptación social, emocional y académica, en este último aspecto es relevante para nosotros tratar de entender cómo se modifica el rendimiento académico según el logro de las habilidades sociales que los jóvenes tengan.

El rendimiento académico se define en el ámbito universitario como un resultado o un logro de aprendizaje y comprensión sobre diferentes materias, alcanzadas por el estudiante. Es decir, como el logro de conocimientos en un área o materia, es evidenciado a través de indicadores numéricos, el cual es expresado mediante calificación en el sistema vigesimal, para áreas de conocimiento determinadas, para contenidos de una determinada asignatura.

En el ambiente educativo superior una de las dimensiones más importantes en el proceso de enseñanza y aprendizaje resulta siendo el rendimiento académico que logra el estudiante en un periodo de estudio. Debemos de tener en cuenta que hay muchos factores que intervienen $y$ muchos de ellos modifican o influyen en el rendimiento académico; se conoce que los factores socioeconómicos, la amplitud de los programas de estudio, los métodos de enseñanza aplicados por los docentes, la falta de una enseñanza personalizada, la información previa con la que cuenta el estudiante y otros como el estado de madurez con la que cuenta el estudiante, son factores que intervienen en el rendimiento académico de los estudiantes (Edel 2003). Por otro lado Lamas (2015) manifiesta en el rendimiento académico intervienen factores que condicionan dicha variables, como es el caso del nivel intelectual, la personalidad, la motivación, las aptitudes, los intereses, los hábitos de estudio, la autoestima o las relaciones docente- estudiante.

Lamas (2015), dice que el propósito del rendimiento académico es alcanzar una meta educativa o un aprendizaje significativo, lo cual resulta siendo un problema que preocupa a todos los involucrados en los procesos de enseñanza y aprendizaje. Se debe de entender el rendimiento académico como un logro complejo de materializar.

Edel en el 2003 manifiesta que existen variables que están relacionadas al rendimiento académico y fracaso escolar, como es el caso de la motivación escolar, el autocontrol del estudiante y las habilidades sociales. Con respecto a esta última diremos que es probablemente el más relacionado. Las habilidades sociales en un estudiante promueven el máximo aprovechamiento de sus capacidades y contribuye a neutralizar los efectos negativos de un ambiente social y familiar desfavorable, mediante las habilidades sociales el estudiante universitario tiene la oportunidad de adquirir técnicas, conocimientos, actitudes y hábitos que le permiten una mejor adaptación al entorno educativo. El estudiante que tiene habilidad social, socializa mejor, tiene un mejor control de su persona y una mejor predisposición a la adquisición de responsabilidades.

Las habilidades sociales se consideran como un conjunto de comportamientos dentro de la persona, que resultan siendo complejos. Las habilidades son competencias que se van adquiriendo o aprendiendo a lo largo de un tiempo de relación con el entorno social, son conductas que se aprenden para alcanzar una meta pre establecida. Se hacen esfuerzos por establecer un instrumento que mida en forma confiable las habilidades sociales, con resultados satisfactorios (Bolsoni \& Loureiro, 2020). 
Las investigaciones sobre habilidades sociales está experimentado un importante auge en estos últimos años, se buscó mediante un estudio comparativo de las habilidades sociales de madres y los indicadores de ansiedad y de comportamiento de niños, obteniéndose tasas altas de problemas de conducta y síntomas depresivos en las madres que mostraron niveles más bajos de habilidades sociales (Assis \& Bolsoni, 2020). En especial teniendo en consideración que en nuestra experiencia personal diaria somos conscientes que pasamos un alto porcentaje de nuestro tiempo en interacciones sociales e interpersonales, debemos de tener en cuenta que tener relaciones sociales positivas permiten un aumento de la autoestima y el bienestar personal. Este contexto de bienestar y mejora de la autoestima es importante cuando los docentes Universitarios interactuamos con nuestros estudiantes en forma cotidiana. García en el 2010, ha demostrado que los alumnos han recibido una adecuada formación en habilidades sociales, que podría mejorar su conducta de relaciones interpersonales, logrando una aceptación social entre sus compañeros.

En la actualidad no damos cuenta y hemos comprobado por la experiencia cotidiana, que la competencia social de los individuos, tiene una contribución importante a sus logros personales, puesto que hoy en día el éxito personal y social parece estar más relacionado con las habilidades sociabilidad y las interpersonales del sujeto.

Se ha sugerido que se debe de entrenar en habilidades sociales en pacientes psiquiátricos, en especial los que tienes situaciones relacionadas con el uso de drogas, con la finalidad de motivar autocontrol y disminución de la agresividad (Limberger et al., 2019). Esta competencia social es una competencia fundamental que ha de tener adquirida todo educador para el desempeño en su experiencia pedagógica cotidiana. En el entorno académico no se aborda el concepto de habilidades sociales, se ha evidenciado que estas se desarrollan de manera natural, no planificada $\mathrm{y}$ en muchos casos de manera insuficiente, esto puede causar un desmedro en el desempeño profesional, en especial en las áreas de las ciencias de la Salud (Carneiro et al., 2020). El objetivo de la presente investigación fue determinar la relación que existe entre las Habilidades Sociales que ha logrado un estudiante y el Rendimiento Académico logrado durante sus estudios en la Escuela profesional de Medicina Humana de la Universidad Andina del Cusco, durante el año 2019

\section{MÉTODOS Y MATERIALES}

Estudio de tipo descriptivo, transversal y relacional, realizado en la Universidad Andina del Cusco. El muestreo fue probabilístico y la muestra estuvo constituida por 201 estudiantes. Para la recolección de los datos los participantes firmaron su consentimiento informado. Se mantuvo el anonimato de los participantes.

Para la selección de las unidades de análisis se tomó en cuenta criterios de selección como que estén matriculados durante el semestre académico, alumnos que deseen formar parte en forma voluntaria en la investigación, que lleven la totalidad de créditos durante el semestre matriculado. 


\section{RESULTADOS}

Tabla $\mathrm{N}^{\circ} 1$. Distribución estadística del promedio ponderado, habilidades sociales y sus dimensiones en estudiantes universitarios de Cusco

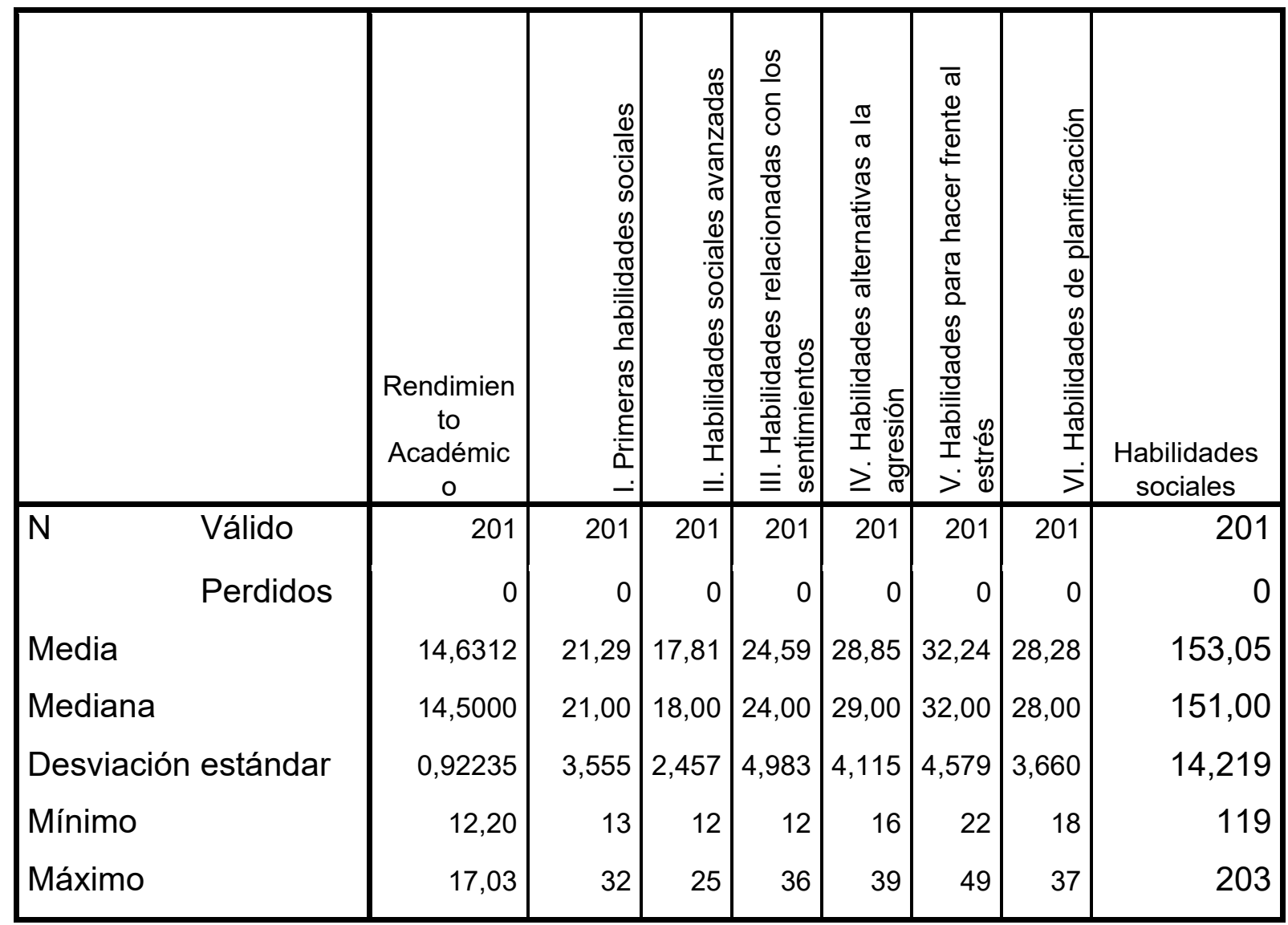

En la presente tabla se puede observar que los estudiantes de medicina humana obtuvieron un promedio ponderado de 16,63 puntos sobre la calificación total de 20 , se registró estudiantes que tuvieron un promedio de 12.20 y estudiantes que tuvieron un promedio de 17,03 . Con respecto a las habilidades sociales se obtuvo un promedio de 153 puntos sobre el total de 250 puntos que se podría obtener, dichos resultados les permitió ser ubicados dentro del eneatipo II, es decir un bajo logro de habilidades sociales en promedio. Se registró estudiantes con un puntaje de 203, permitiéndoles ubicarse en el eneatipo 7 con un logro normal de habilidades sociales. Pero también se registraron estudiantes que obtuvieron 119 puntos que les permitió ser ubicados en el eneatipo 1, es decir un logro deficiente de habilidades sociales. 


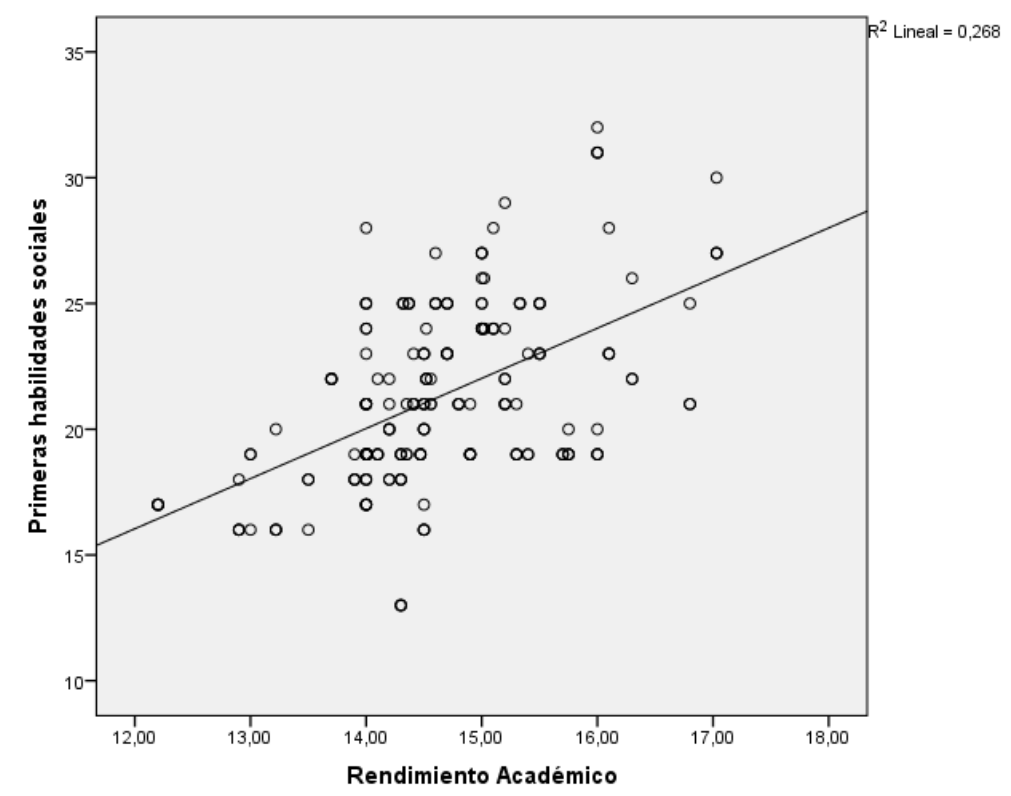

Grafico 01 Relación de las primeras habilidades sociales con el rendimiento académico de los estudiantes universitarios de Cusco

En la gráfica número 01 se puede observar que existe una relación directamente proporcional entre las Primeras habilidades sociales y el rendimiento académico. Es decir a un mayor nivel de saber escuchar, iniciar una conversación, mantener una conversación, formular una pregunta, dar las gracias, presentarse, presentar a otras personas y hacer un cumplido, existe un mejor rendimiento académico en los estudiantes de Medicina Humana de la Universidad Andina del Cusco. A la prueba estadística correlacional de Pearson se obtuvo un valor $r=0,517$. Existiendo una moderada correlación entre las variables, con un factor de determinación de probabilidad de causa efecto de $26,8 \%$.

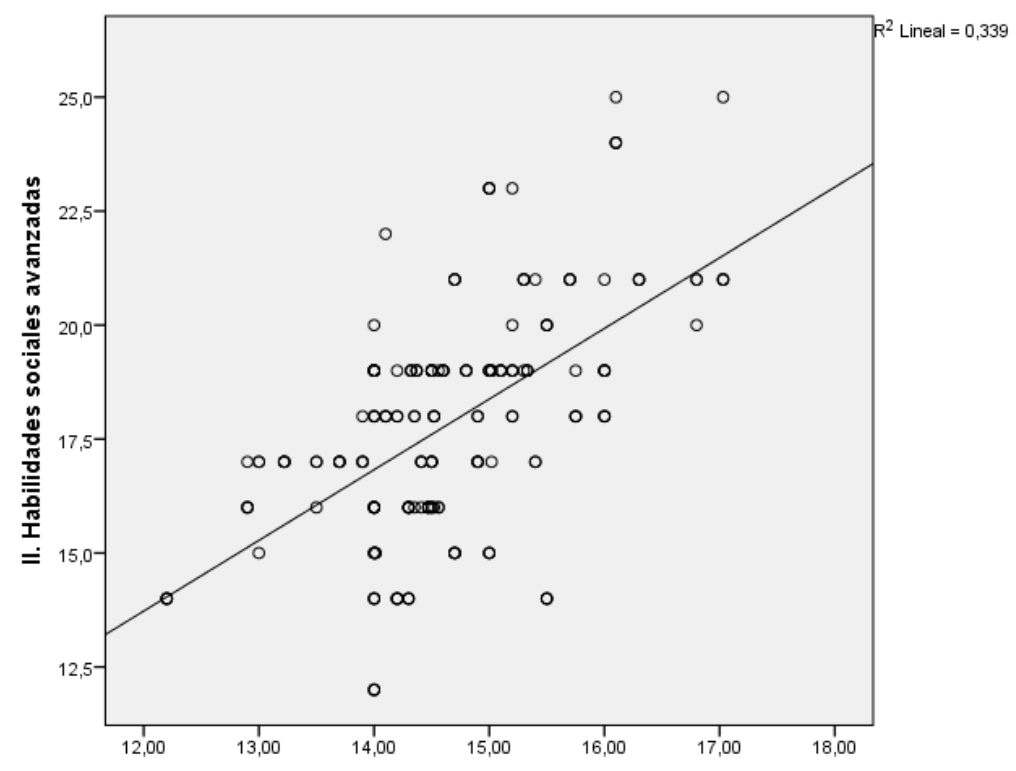

Grafico 2 Relación de las habilidades sociales avanzadas con el rendimiento académico de los estudiantes universitarios de Cusco 
En la gráfica número 02 se puede observar que existe una relación directamente proporcional entre las Habilidades sociales avanzadas y el rendimiento académico. Es decir a un mayor nivel de relacionarse satisfactoriamente en el entorno, como es el caso de pedir ayuda, participar, dar instrucciones, seguir instrucciones, disculparse, convencer a los demás, existe un mejor rendi- miento académico en los estudiantes de Medicina Humana de la Universidad Andina del Cusco. A la prueba estadística correlacional de Pearson se obtuvo un valor $r=0,582$. Existiendo una moderada correlación entre las variables, con un factor de determinación de probabilidad de causa efecto de $33,9 \%$.

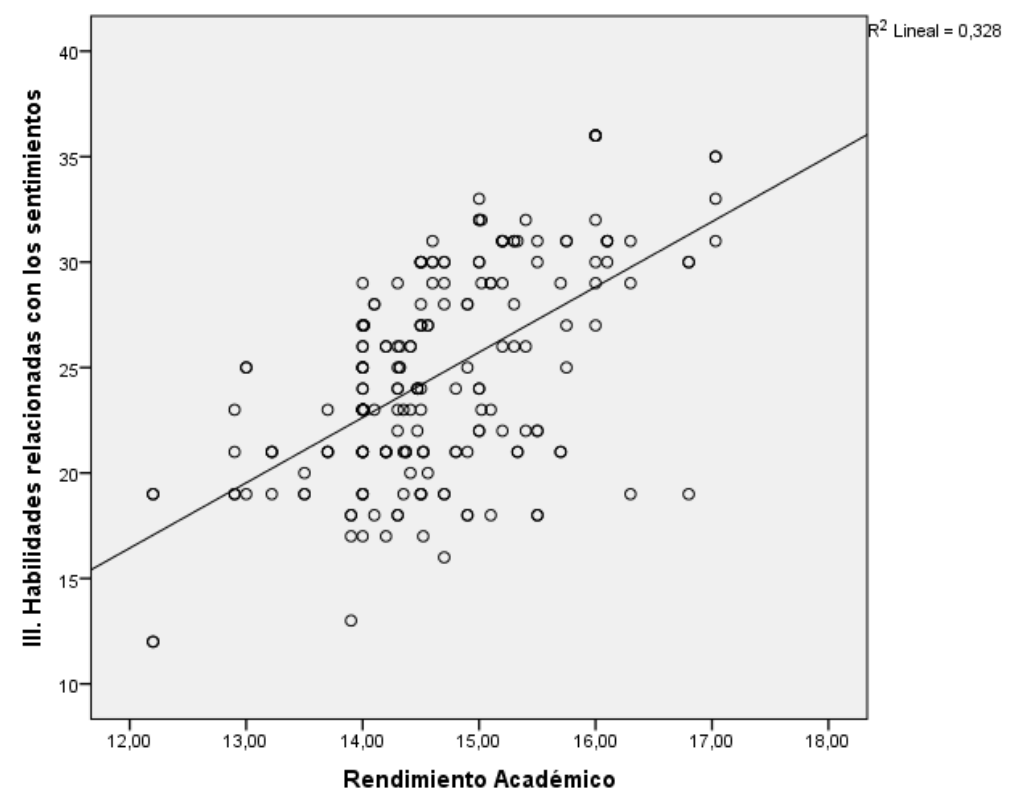

\section{Grafico 3 Relación de las habilidades relacionadas con los sentimientos con el ren- dimiento académico de los estudiantes universitarios de Cusco}

En la gráfica número 03 se puede observar que existe una relación directamente proporcional entre las Habilidades relacionadas con los sentimientos y el rendimiento académico. Es decir a un mayor nivel de conocer los propios sentimientos, expresar los sentimientos, comprender los sentimientos de los demás, enfadarse con el enfado de otro, expresar afecto, resolver el miedo, autorrecompensarse, existe un mejor rendimiento académico en los estudiantes de Medicina Humana de la Universidad Andina del Cusco. A la prueba estadística correlacional de Pearson se obtuvo un valor $r=0,573$. Existiendo una moderada correlación entre las variables, con un factor de determinación de probabilidad de causa efecto de $32,8 \%$. 


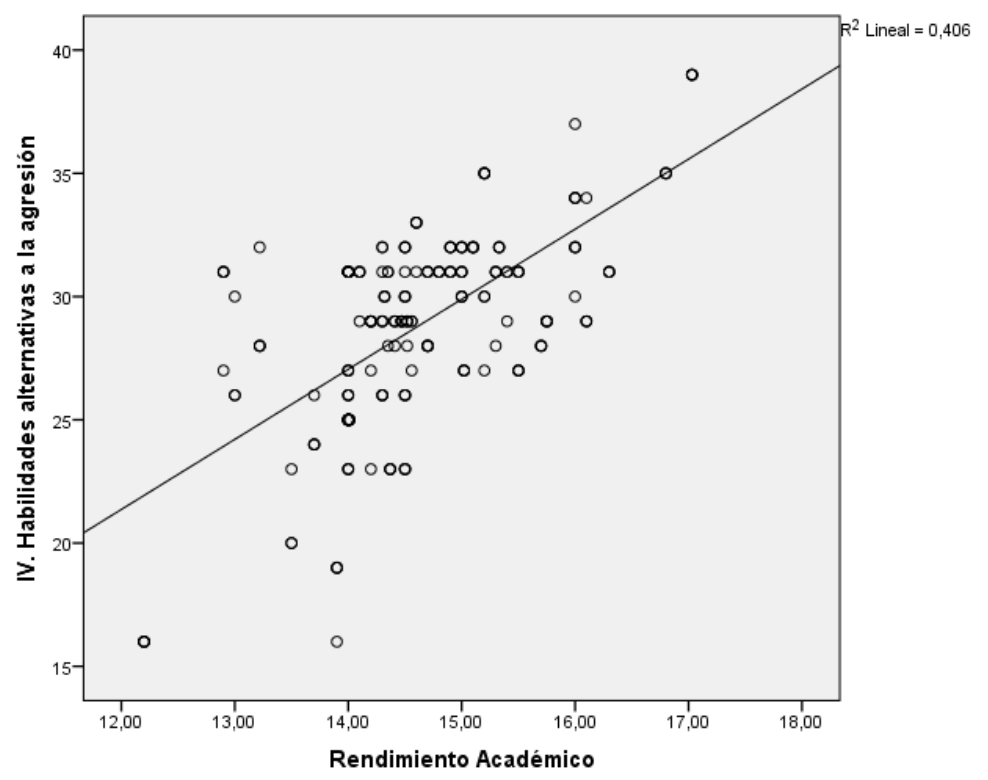

\section{Grafico 4 Relación de las habilidades alternativas a la agresión con el rendimiento académico de los estudiantes universitarios de Cusco}

En la gráfica número 04 se puede observar que existe una relación directamente proporcional entre las Habilidades alternativas a la agresión y el rendimiento académico. Es decir a un mayor nivel de establecimiento de objetivos y toma de decisiones y resolución de conflictos, existe un mejor rendimiento académico en los estudiantes de Medicina Humana de la Universidad Andina del Cusco. A la prueba estadística correlacional de Pearson se obtuvo un valor $r=0,637$. Existiendo una moderada correlación entre las variables, con un factor de determinación de probabilidad de causa efecto de $40,6 \%$.

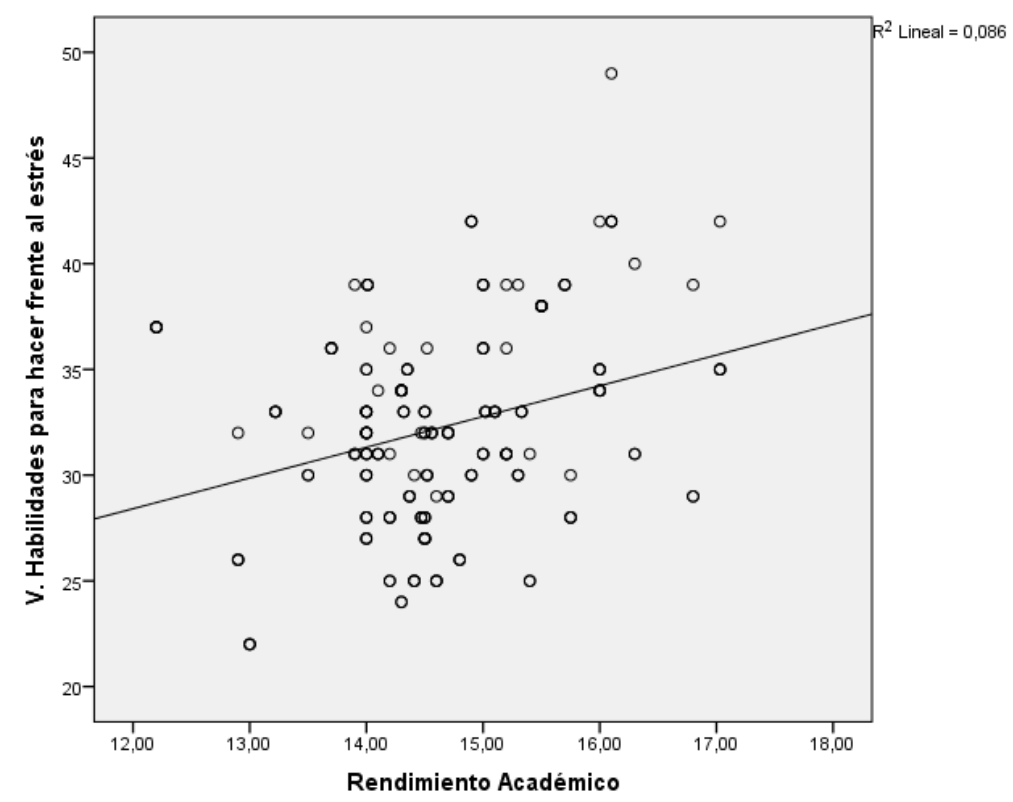

Grafico 5 Relación de las habilidades para hacer frente al estrés con el rendimiento académico de los estudiantes universitarios de Cusco 
En la gráfica número 05 se puede observar que existe una relación directamente proporcional entre las Habilidades alternativas a la agresión y el rendimiento académico. Es decir a un mayor nivel de establecimiento de objetivos y toma de decisiones y resolución de conflictos, existe un mejor rendimiento académico en los estudiantes de Medicina Humana de la Universidad Andina del Cusco. A la prueba estadística correlacional de Pearson se obtuvo un valor $r=0,293$. Existiendo una baja correlación entre las variables, con un factor de determinación de probabilidad de causa efecto de $8,6 \%$.

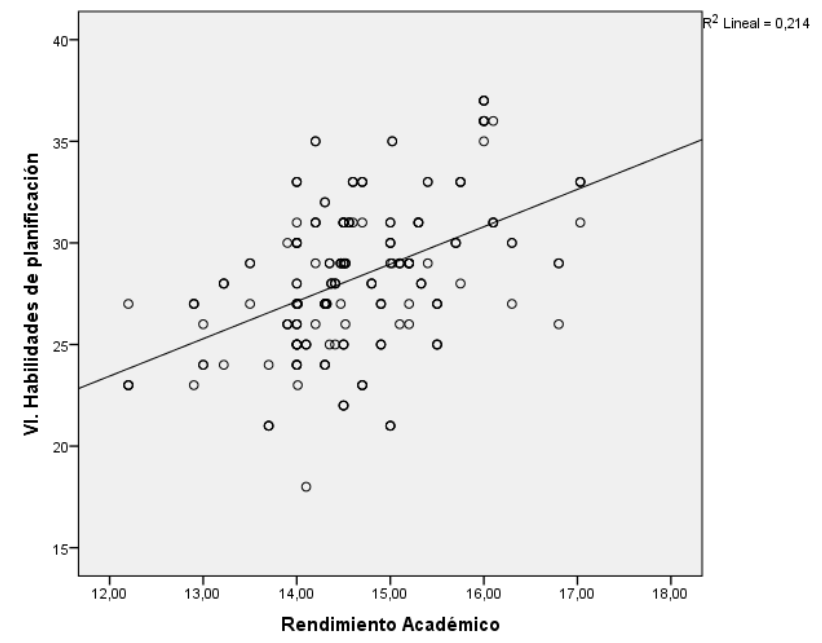

\section{Grafico 6 Relación de las habilidades de planificación con el rendimiento académico de los estudiantes universitarios de Cusco}

En la gráfica número 07 se puede observar que existe una relación directamente proporcional entre las habilidades de planificación y el rendimiento académico. Es decir a un mayor nivel de desarrollo de autocontrol y empatía al momento de situaciones de enfado, existe un mejor rendimiento académico en los estudiantes de Medici- na Humana de la Universidad Andina del Cusco. A la prueba estadística correlacional de Pearson se obtuvo un valor $r=0,463$. Existiendo una moderada correlación entre las variables, con un factor de determinación de probabilidad de causa efecto de $21,4 \%$.

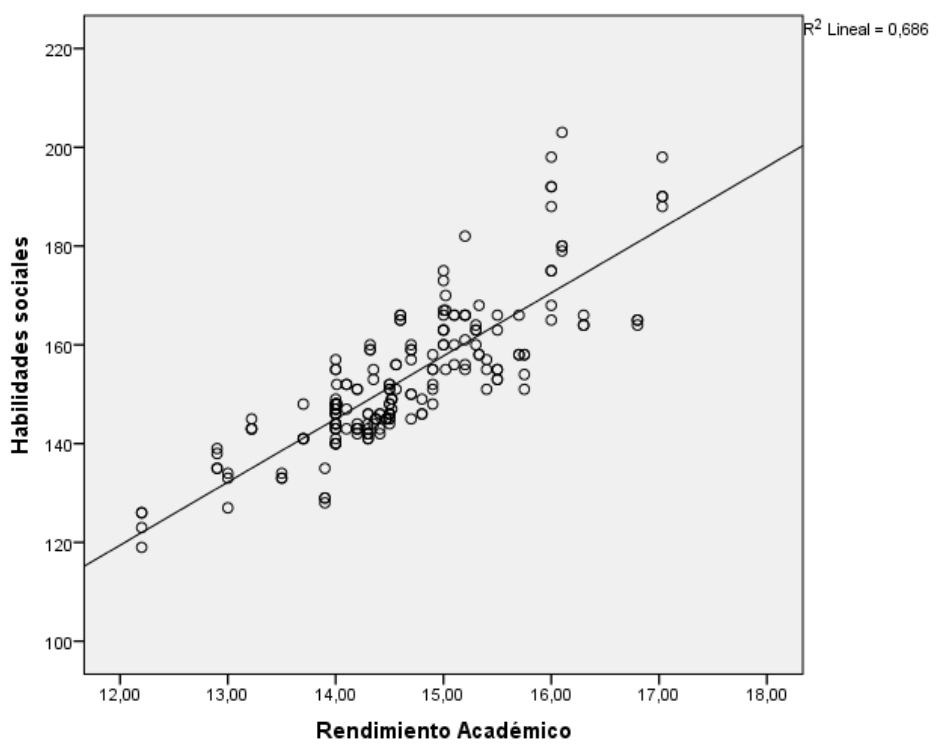

Grafico 6 Relación de las habilidades de planificación con el rendimiento académico de los estudiantes universitarios de Cusco 
En la gráfica número 07 se puede observar que existe una relación directamente proporcional entre las habilidades sociales y el rendimiento académico. Es decir a un mayor nivel de destreza para actuar socialmente, existe un mejor rendimiento académico en los estudiantes de Medicina Humana de la Universidad Andina del Cusco. A la prueba estadística correlacional de Pearson se obtuvo un valor $r=0,829$. Existiendo una muy buena correlación entre las variables, con un factor de determinación de probabilidad de causa efecto de $68,6 \%$.

\section{DISCUSIÓN}

Las habilidades sociales es un conjunto de conductas emitidas en un contexto interpersonal que expresa los sentimientos, actitudes, deseos, opiniones o derechos de un modo adecuado a la situación inmediata, respetando esas conductas en los demás. La habilidad social le permite al estudiante generalmente resolver los problemas inmediatos de la situación mientras minimiza la probabilidad de futuros problemas. Los contextos por los que puede atravesar el estudiante en su vida universitaria, le exige un proceso permanente de adaptación y empatía con sus compañeros. Existen profesiones que exigen un logro alto de habilidades sociales, entre ellas se encuentra las Medicina Humana, es desde la formación universitaria que el estudiante debe de logara y desarrollar esta capacidad de relación, que le permita tener éxito en los tratamiento con sus pacientes. Nosotros encontramos habilidades sociales poco desarrolladas en nuestro estudiantes de medicina humana, lo cual se puede deber a la falta de interés y conocimiento en los planes de estudio o curriculares en la carrera. Se obtuvo un bajo logro en los jóvenes. Por otro lado la inteligencia emocional que está estrechamente relacionado a las habilidades sociales permite al estudiante mantener sus relaciones con sus similares, manteniendo un ambiente de armonía y convivencia, las habilidades es un elemento importante que debe de modificar y desarrollar en forma constante (Guzmán, 2018). Las habilidades sociales son conductas aprendidas que se necesita para el desempeño profesional a nivel personal e interpersonal Carneiro et al. (2020) evaluó las habilidades sociales al inicio y fin de un curso, obteniendo un promedio de 135,35.

El rendimiento académico se define en el ámbito universitario como un resultado o un logro de aprendizaje y comprensión sobre diferentes materias, alcanzadas por el estudiante. Es decir como el nivel demostrado de conocimientos en un área o materia, evidenciado a través de indicadores cuantitativos, usualmente expresados mediante calificación ponderada en el sistema vigesimal y, bajo el supuesto que es un "grupo social calificado" el que fija los rangos de aprobación, para áreas de conocimiento determinadas, para contenidos específicos o para Asignaturas. En nuestro estudio se obtuvo un rendimiento académico de aprobación con un promedio ponderado de 14,63 . Resultados similares a los obtenido por Gonzales et al. (2016) quienes obtuvieron un promedio de 13.82 , resultados que difieren con lo obtenido por García (2005) quienes obtuvieron un alto porcentaje de rendimiento académico bajo, es decir con notas desaprobatorias.

En el estudiante en formación se pueden generar emociones intensas como la ansiedad, que puede interferir en su capacidad cognitiva, pero por otro lado las emociones positivas pueden aumentar su capacidad creativa, es así que el logro de habilidades sociales genera un mejor rendimiento académico (Laudadío \& Mazzitelli, 2019). Po otro lado debemos de entender que la educación se da en un entorno social. Para que los estudiantes de educación superior puedan llegar a tener un resultado satisfactorio a nivel académico es necesario desarrollar competencias sociales que le permitan adaptarse al contexto de la educación superior. Debemos de entender que los estudiantes se adapten al entorno académico, resuelvan sus problemas de conflicto interpersonal, tomen decisiones asertivas con sus compañeros y docentes y mantener un ambiente amistoso y fraterno. En nuestro estudio se evidencio una relación directamente proporcional entre el rendimiento académico y el logro de 
habilidades sociales, con un facto de determinación del 68,6\% es decir existe la probabilidad de que a un mayor logro de habilidades sociales el estudiante tenga mejor rendimiento académico. Carneiro et al. (2020) evidencio que la participación en actividades académicas pueden afectar el desarrollo de las habilidades sociales, resultados similares a lo obtenido por nuestro estudio. Así como Oyarzun et al. (2012) afirman que las habilidades sociales positivas están asociadas a un mejor rendimiento académico, en especial entre el sexo femenino. Resultados que es compartido por Cadoche, L. (2009), quien hizo una análisis ampliado el tema, evidenciando que existen otros factores que influyen en le rendimiento académico del estudiante. Es así que también Dapelo et al. (2013), Gonzales et al. (2016) y Piña R. (2010) quienes concluyeron que el logro de las habilidades sociales se asocia al logro de la competencia académica.

\section{CONCLUSIONES}

Primera.- el rendimiento académico logrado es de aprobación y las habilidades sociales que poseen es de un bajo logro en los estudiantes universitarios del Cusco.

Segunda.- existe moderada correlación directamente proporcional entre las Primeras habilidades sociales que poseen los estudiantes universitarios y su rendimiento académico.

Tercera.- existe moderada correlación directamente proporcional entre las Habilidades sociales avanzadas que poseen los estudiantes universitarios y el rendimiento académico.

Cuarta.- existe moderada correlación directamente proporcional entre las habilidades relacionadas con los sentimientos que poseen los estudiantes universitarios y el rendimiento académico.

Quinta.- existe buena correlación directamente proporcional entre las Habilidades alternativas a la agresión que poseen los estudiantes universitarios y el rendimiento académico.
Sexta.- existe baja correlación directamente proporcional entre las habilidades para hacer frente al estrés que poseen los estudiantes universitarios y el rendimiento académico.

Séptima.- existe moderada correlación directamente proporcional entre las habilidades de planificación que poseen los estudiantes universitarios y el rendimiento académico.

Octava.- existe muy buena correlación directamente proporcional entre las Habilidades Sociales que poseen los estudiantes universitarios y el rendimiento académico.

\section{REFERENCIAS BIBLIOGRAFICAS}

Assis, R. P., \& Bolsoni, A. T. (2020). Educational Social Skills and Repertoire of Children Differentiated by Behavior and Sex. Paidéia (Ribeirão Preto), 30(1).

Bolsoni, A. T., \& Loureiro, S. R. (2020). Evidence of validity for Socially Skillful Responses Questionnaires-SSRQ-Teachers and SSRQ-Parents. Psico-USF, 25(1), 155-170.

Carneiro, F., Matias, L. B., \& Moreira, J. M. (2020). Habilidades sociales de estudiantes de Enfermería e Psicología. Ciencias Psicológicas, 14(1), 1-11.

Guzmán, M. del C. (2018). DESARROLLO DE HABILIDADES SOCIALES A TRAVÉS DE ACTIVIDADES LÚDICAS EN LOS NIÑOS DE 3 AÑOS EN EL CENTRO INFANTIL CUMBAYA VALLEY. Conrado, 14(64), 153-156.

Laudadío, J., \& Mazzitelli, C. (2019). Formación del profesorado: Estilos de enseñanza y habilidades emocionales. Revista mexicana de investigación educativa, 24(82), 853-869.

Limberger, J., dos Santos, B. S., Ribeiro, E., \& Andretta, I. (2019). Autocontrol de la agresividad de las usuarias de crack. Ciencias Psicológicas, 13(2), 173-184. 